\title{
Thermal Conductivity of Gases. I. The Coaxial Cylinder Cell ${ }^{1}$
}

\section{Leslie A. Guildner}

(April 13, 1962)

\begin{abstract}
By combining appropriate geometric configuration and mathematical analysis with improved measuring techniques, the cell constant of a coaxial cylinder thermal conductivity cell was determined within 0.1 percent.

An analysis of the rate of heat transfer in such a cell showed a way to treat the data so that the error contribution of experimental deviations from idealized conditions is kept small. The principal considerations are:

1. That heat transport by convection is significantly large in a dense gas. This transport was analyzed mathematically from basic principles. The agreement of experimental results with the analysis indicated that the expressions are valid and that the convective heat transport could be accounted for with little more error than was involved in the precision of the heat transfer measurements.

2. That the heat transfer in a vacuum corresponds to the heat transfer by radiation and solid contacts in the presence of a gas. The uncertainty was that associated with the accuracy of determining the vacuum values.

3. That other effects were small enough to be computed and corrected for without increasing the uncertainty of the values of the thermal conductivity.
\end{abstract}

\section{Introduction}

The coaxial cylinder thermal conductivity cell, with large diameter of inner cylinder relative to the conductivity gap width, is one of the forms of apparatus often used to determine the thermal conductivity of gases. Over a period of several years, refinements were made in the design of a cell, in the analysis of heat transfer, in measurement techniques and in the treatment of the data. It is the purpose of this paper to set forth a summary of considerations which are applicable to measurements with a cell of this general type.

The heat guards at the ends of a coaxial cylinder cell can be designed so that the geometric form of the conductivity gap is simple. It is then possible to make a reliable mathematical analysis of the heat transfer by conduction. Consistent with a nearly exact mathematical analysis, special techniques were used for measuring the cell dimensions with improved accuracy.

At sufficiently high gas densities, convective heat transport becomes significant in a coaxial cylinder cell. When the axis of the cell is vertical, the heat transport by convection can be analyzed from basic principles. This analysis indicates the proper measurements to be made and the required treatment of the data.

Other effects which should be accounted forasymmetry of the heat flow, radiation from the emitter and conduction through the mounting pins, and the temperature gradient in the body of the metal-are considered. The significance of these effects will be clearer after understanding some of the details of a cell, which will be described in the next section.

${ }^{1}$ This work was performed at the Massachusetts Institute of Technology and sponsored by Project SQUID, which is supported by the Office of Naval Research, Department of the Navy, under contract Nonr 1858(25) NR-098-038. Reproduction in full or in part is permitted for use of the United States Government.

$637358-62-5$

\section{Apparatus}

The cell shown in figure 1 was made of silver. It consisted of an emitter $E M$, surrounded by a receiver $R E$. The emitter was located by seven Pyrex pins

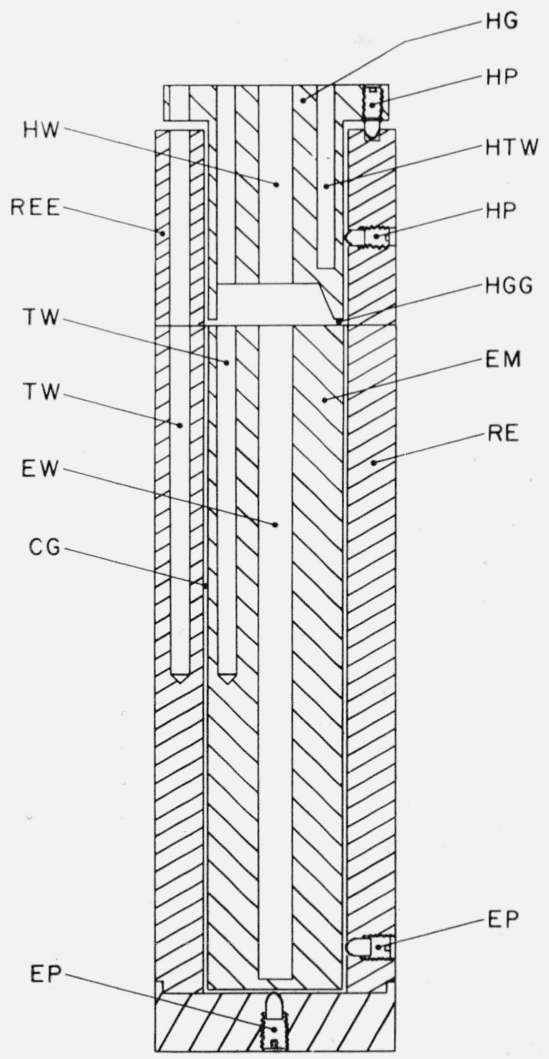

FiguRE 1. Vertical cross section of coaxial cylinder cell. 
$E P$, one on the axis at the bottom, three spaced uniformly around the receiver near the bottom, and three (not shown) similarly spaced near the top. Electrical energy was supplied to the emitter by a heater in the heater well $E W$. The heater was made from Nichrome ribbon uniformly wound on a machined Grade "A" "Lava" form. The temperature or the temperature difference was measured by thermocouples inserted in the thermocouple wells $T W$ of the emitter and receiver. The junctions were at two levels in the receiver and emitter, one level being midway along the emitter close to the bottom of the wells, and the other level just below the top of the emitter. The thermocouples were installed between the wall of the well and an inserted rod of silver. The thermocouple wires were insulated by thin sheets of mica, and they were packed tightly in the thermocouple wells.

A heat guard $H G$ provided a continuation of the conductivity gap $C G$. It was positioned by six Pyrex pins $H P$, three spaced uniformly around the side and three around the top. The top pins were adjusted so that the width of the heat guard gap $H G G$ was equal to the width of the conductivity gap. The heat guard temperature was maintained as close as possible to the temperature of the emitter, by introducing electrical energy from a heater in the heat guard heater well $H W$. The temperature was measured by a thermocouple whose junction was placed near the bottom of the heat guard thermocouple well $H T W$.

The leads from the emitter heater and the emitter and receiver thermocouples passed through the heat guard or the corresponding portion of the receiver, the receiver extension $R E E$. (The receiver extension surrounding the heat guard was separate to facilitate assembly.) The thermocouple leads were insulated with mica and in order to improve heat transfer were held against the walls of the thermocouple wells, by means of a split rod of silver, which was wedge shaped.

The mounting pins were made in three parts: a Pyrex rod, $3 \mathrm{~mm}$ in diameter with a $60^{\circ}$ included angle conical point, a pure aluminum holder, and a silver screw. The lengths of Pyrex and aluminum were chosen such that their composite expansion was close to that of silver over the temperature range 0 to $400{ }^{\circ} \mathrm{C}$.

The dimensions of this cell, although not essential to the discussion in this paper, are used for illustration, and were approximately:

\section{Conductivity gap \\ Heat guard length \\ Emitter diameter \\ Emitter length \\ $0.068 \mathrm{~cm}$ \\ $3.8 \mathrm{~cm}$ \\ $2.2 \mathrm{~cm}$ \\ $11.4 \mathrm{~cm}$.}

The dimensions should be chosen so that $\Delta r r \bar{r} \leq 0.1$. The length of the emitter should be sufficient for 90 or 95 percent of the heat transfer to take place radially, and the length of the heat guard should be enough to reduce unaccountable heat loss from the emitter satisfactorily. The choice of the size of the conductivity gap represents a compromise. On the one hand, a narrow gap makes the ratio of the heat transfer by conduction large relative to the heat transfer by radiation and also very effectively makes the relative heat transport by convection small. On the other hand, when the gap is made too narrow, the uncertainty in the value of the cell constant is increased. The choice of dimensions will depend upon the objectives of the investigation.

\section{The Cell Constant}

\subsection{Mathematical Treatment}

We can obtain the cell constant from the assumption that the rate of heat transfer, $\dot{q}$, is proportional to the temperature gradient and the surface area. In the steady state, we can integrate the temperature gradient over the region of heat flow, so that $q=C K \Delta t$, where $C$ is the cell constant, $K$ is the thermal conductivity, and $\Delta t$ is the temperature difference across the region in the steady state condition.

The principal terms of the cell constant can be evaluated by considering the radial heat flow across a section of length $l$ of an infinite coaxial cylinder and the linear heat flow across a circular section of radius $r_{1}$ of two infinite parallel plates at the bottom. The determination of an accurate cell constant requires that recognition be made of the additional heat flow which takes place at the heat guard gap and at the bottom corner of the emitter. If both the conductivity gap, $\Delta r$, and the heat guard gap are small compared to the radius of the emitter, $r_{1}$, the conductivity gap and adjacent portion of the heat guard gap can be treated as if planar. In figure 2, the relaxation solution for the gas isotherms near the heat guard shows that the perturbing effect of the gap has practically vanished within two gap widths in any direction. Therefore, it is proper to employ the Schwarz-Christoffel transformation which is valid for an infinite cell. The transformations for both the heat guard gap and the bottom corner are given in Carslaw and Jaeger, [1] ${ }^{2}$ pp. 444 and 445. In an analogous manner to the solution for the bottom corner given on pp. 453-454, the correction terms for the heat guard gap may be found. On the assumption that the heat flow over the length of the cell takes place as if unperturbed, the deviations may be combined as a factor times half the heat guard gap, which is to be added to the length of the cell. If the heat guard gap is equal to the conductivity gap, $\Delta r$, the added length is ${ }^{3}$

$$
c_{2}=0.923 \Delta r / 2 \text {. }
$$

The bottom corner of the emitter adds a term to the cell constant of $2 \pi r_{1} \times 0.559$ for equal conductivity gaps on the sides and bottom. Thus, the total rate of heat transfer by gas conduction is

$$
\dot{q}=K\left[\frac{2 \pi\left(l+c_{2}\right)}{\ln r_{2} / r_{1}}+\frac{\pi r_{1}^{2}}{\Delta r}+2 \pi r_{1} \times 0.559\right] \Delta t
$$

where $r_{2}$ is the inner radius of the receiver. The

${ }^{2}$ Figures in brackets indicate the literature references at the end of this paper. 3 See appendix 1 . 


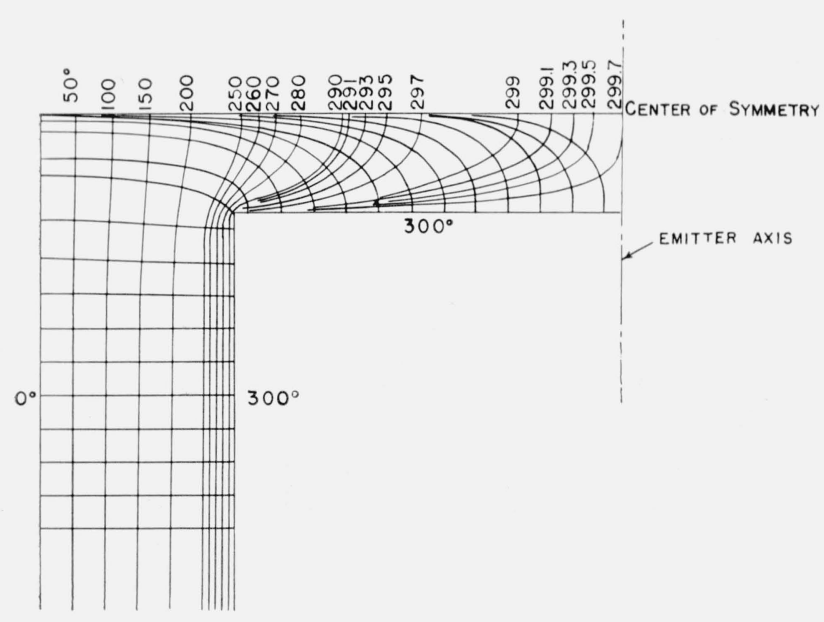

Figure 2. Relaxation solution of the heat guard gap.

cell constant is

$$
C=\pi\left[\frac{2\left(l+c_{2}\right)}{\ln r_{2} / r_{1}}+\frac{r_{1}^{2}}{\Delta r}+1.118 r_{1}\right] .
$$

From eq (2), we find 99.4 percent of the heat transfer from the cell illustrated in figure 1 is accounted for by the two principal parts: 94.9 percent by radial heat flow, 4.5 percent by linear heat flow. The transfer from the heat guard gap amounts to 0.25 percent, and the transfer from the bottom corner amounts to 0.33 percent.

\subsection{Measurement of Receiver and Emitter Radii}

Both to increase the flow of heat by conduction and to reduce the heat transferred by convection, the conductivity gap should be kept as small as will permit the accurate determination of the cell constant. For the case of $\frac{\Delta r}{r_{1}} \ll 1$, the rate of heat transfer by conduction from a section of length $l$ of an infinite coaxial cylinder is $\dot{q}=\frac{2 \pi \bar{r} K l \Delta t}{\Delta r}$ within a relative error of $\frac{1}{3}\left(\frac{\Delta r}{2 \bar{r}}\right)^{2}$, or 0.03 percent for $2 \bar{r}=2.2$ $\mathrm{cm}$ and $\Delta r=0.068 \mathrm{~cm}$. The equation serves to emphasize that for the same error in the cell constant two orders of higher accuracy of length measurements are required to determine the conductivity gap, than the radii themselves.

The measurements of the two radii were made with a super-micrometer as measurements of two outer diameters, the one directly, the other by use of a "falling probe." If a "probe" is allowed to fall by its own weight, as shown in figure 3 , the gap between the probe and the outer cylinder can be calculated from the air flow [2]. In an annulus formed by two coaxial cylinders of radius $r_{p}$ and $r_{2}$, length $L$, and gap $m=r_{2}-r_{p}$, the volume of gas of viscosity $\eta$ flowing in time $\theta$ with a difference of pressure

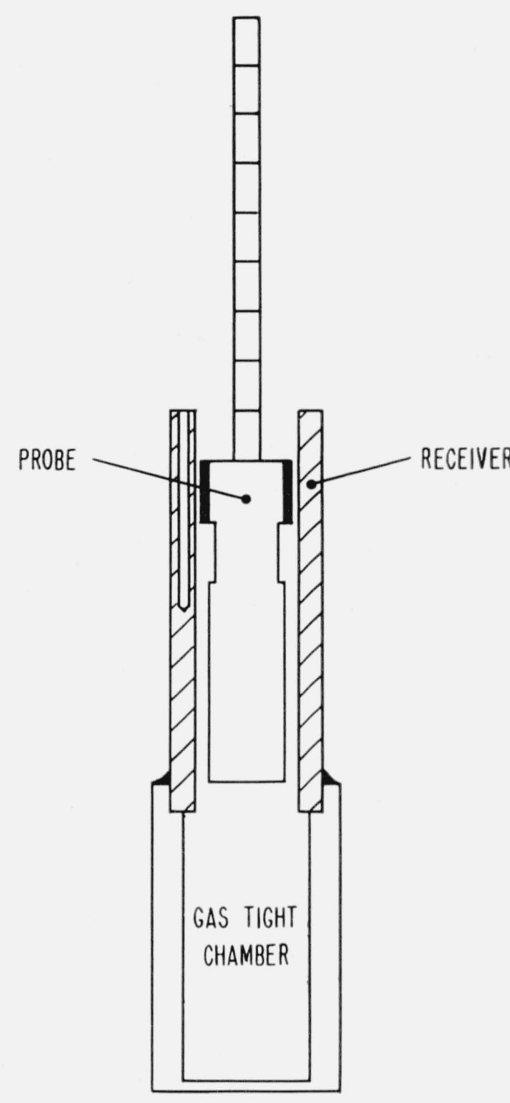

FiguRE 3. Falling probe device for inner diameter measurement.

$\Delta p=p_{1}-p_{2}$ and average pressure $\bar{p}$ can be expressed within a relative error of $\frac{m^{2}}{60 r_{p}^{2}}$ as

$$
\Delta V=\frac{\pi \bar{p} \Delta p g \bar{r} m^{3} \theta}{6 \eta p_{2} L}
$$

$\bar{r}=\left(r_{p}+r_{2}\right) / 2$ and $p_{2}$ is the atmospheric pressure.

With the volume expressed as the probe displacement

$$
m^{3}=\frac{6 \eta p_{2} r_{2}^{2} \Delta l L}{g \bar{p} \Delta p \bar{r} \theta}
$$

where $g$ is the gravitational constant and $\Delta l$ is the distance of fall in time $\theta$.

The average inner radius of the receiver is determined by adding the gap, $m$, to the radius of the probe, $r_{p}$. The determination of the gap, $m$, was precise within $2.5 \times 10^{-5} \mathrm{~cm}$.

The measurement of $2 r_{p}$ was precise within $2.5 \times 10^{-5} \mathrm{~cm}$, as well as $2 r_{1}$, the outer diameter of the emitter. The sum of $m+r_{p}$ for varying $m$ was consistent within $2.5 \times 10^{-5} \mathrm{~cm}$. Thus with differences of radius between receiver and emitter as small as $0.06 \mathrm{~cm}$, the length of $\Delta r$ was determined to an accuracy within 0.1 percent. 


\section{Convection}

In general, the hydrodynamical equation for nonturbulent flow of a fluid of velocity $\bar{r}$, viscosity $\eta$, pressure $p$, and density $\rho$ is (div $\eta \operatorname{grad}) \bar{v}=\operatorname{grad}$ $p-\rho g$, where $g$ is the acceleration of gravity [3]. For two infinite vertical plates at uniform temperatures $t_{0}-\Delta t / 2$ and $t_{0}+\Delta t / 2$, the equation reduces to

$$
\eta \frac{d^{2} v_{z}(x)}{d x^{2}}=(\rho-\bar{\rho}) g \text {. }
$$

The axes are taken with the origin at the midpoint between the planes. The $x$-axis is normal to the planes, the $y$-axis is horizontal, and the $z$-axis is vertical. The average density, $\bar{\rho}$ is the density at $x=0$.

If the separation of the planes is $\Delta r, t(x)=t_{0}+\frac{x \Delta t}{\Delta r}$ and

$$
\rho=\bar{\rho}\left[1-\alpha\left(t-t_{0}\right)\right]=\bar{\rho}-\frac{\bar{\rho} \alpha x \Delta t}{\Delta r} \text {, where } \alpha=-\frac{1}{\rho}\left(\frac{\partial \rho}{\partial t}\right)_{p} .
$$

Since $v=0$ when $x=-\frac{\Delta r}{2}$ and $x=\frac{\Delta r}{2}$,

$$
v(x)=\frac{\alpha \bar{\rho} g \Delta t x}{6 \bar{\eta} \Delta r}\left[\Delta r^{2} / 4-x^{2}\right]
$$

In a closed system, the circulation takes place as a single stream, reversing direction at the top and bottom. Conservation of mass requires a term second order in $\Delta t$ which does not affect the heat transport by more than 1 percent for actual measurement, hence it was neglected.

The heat transported per unit horizontal distance is

$$
\dot{q}=c_{p} \int_{-\Delta / r 2}^{\Delta r / 2} v(x) \rho(x) \Delta t(x) d x=\frac{\Delta r^{3} g}{720} \frac{\alpha \rho^{2} c_{p} \Delta t^{2}}{\eta}
$$

where $c_{p}$ is the specific heat capacity and the average density is indicated without the bar. The horizontal distance in the cell is very nearly $2 \pi \bar{r}$. Then the rate of heat transport per degree is

$$
\dot{q} / \Delta t=\frac{2 \pi \bar{r} \Delta r^{3} g}{720} \frac{\alpha \rho^{2} c_{p} \Delta t}{\eta} .
$$

If the total heat transfer is studied as a "conductivity," $\dot{q} / C \Delta t$, a portion is due to convection, which may be written as

$$
K_{\mathrm{conv}}=\frac{\dot{q}_{\mathrm{conv}}}{C \Delta t}=\frac{2.6 \times 10^{-6} \alpha \rho^{2} c_{p} \Delta t}{\eta} .
$$

The value $2.6 \times 10^{-6}$ combines the constants of eq (7) and the value of the cell constant $C$ for the cell of figure 1 .

The neglect of terms of higher order in $\Delta t$ for $\rho(x)$ affects $K_{\text {conv }}$ detectibly near the critical point of $\mathrm{CO}_{2}$. When $\Delta t<5 \mathrm{deg} \mathrm{C}$, these terms need not be considered for other measurements to be reported.
Engineering investigations of convection have been analyzed by use of several correlating functions. It may seem at times as if serious discrepancies exist when the correlation is extended to inappropriate conditions. Let us consider three dimensionless quantities:

(1) The Grashof-Prandtl product

$$
\mathrm{Gr} \cdot \operatorname{Pr}=\frac{g(\Delta r)^{3} \alpha \rho^{2} c_{p} \Delta t}{\eta K}
$$

(2) The Grashof number

$$
\mathrm{Gr}=\frac{g(\Delta r)^{3} \alpha \rho^{2} \Delta t}{\eta^{2}}
$$

(3) The Reynolds number

$$
\operatorname{Re}=\frac{\rho \bar{v} x_{0}}{\eta}
$$

where the av erage velocity $\bar{v}=\frac{(\Delta r)^{2} \alpha \rho g \Delta t}{192 \eta}$ and $x_{0}=\Delta r / 2$.

Then

$$
\mathrm{Re}=\frac{g(\Delta r)^{3} \alpha \rho^{2} \Delta t}{384 \eta^{2}}
$$

Thus the Grashof number and the Reynolds number are functionally the same for a gas in a vertical gap. Within the range of pressure and temperature such that the Eucken factor, $K / \eta c_{v}$, is a constant and that $\gamma=\mathrm{c}_{\mathrm{p}} / c_{v}$ is a constant, the Grashof-Prandtl product and the Reynolds number will serve equally well for the correlation of laminar convective effects.

The ratio of $K_{\text {conv }}$ to $K_{\text {aas }}$ can be found by dividing eq (8) by $K_{\text {gas }}$, and is functionally $(\Delta r / l)$ (Gr.Pr). It can be seen from eq $(8) / K_{\text {gas }}$ that the criterion of Kraussold, that convection is insignificant ${ }^{4}$ for $\mathrm{Gr} \cdot \operatorname{Pr}<1000$, leads to different error limits depending upon $\Delta r / l$. For the cell of figure $1, \mathrm{Gr} \cdot \operatorname{Pr}=1000$ involves a relative heat transfer by convection of $8 \times 10^{-3}$.

Representing a ratio of the heat transport of convection to conduction, Gr.Pr is a logical correlating function for heat transport by laminar convection. Over the range of variables that eq (9)/eq (10) is a constant, either will serve as a criterion for the initiation of turbulent convection. Near the critical point, however, $c_{\mathrm{p}} \rightarrow \infty$ and $\gamma \rightarrow \infty$. Since the Eucken factor does not vary greatly, Gr.Pr can become enormous while the Reynolds number remains small. The Reynolds number depends upon the velocity resistance per se, and it is the fundamental quantity, not Gr.Pr, which should be used as the criterion for initiation of turbulent flow.

The purpose of this section is to demonstrate how the data can be treated to give the heat transfer by thermal conduction alone. Measurements must be made under conditions of laminar flow. For $\mathrm{CO}_{2}$, Onsager and Watson [4] have shown that with

4 Worse yet is the statement that convection does not exist for $\mathrm{Gr} \cdot \mathrm{Pr}<1,000$ The author hopes this idea will take its place with the phlogiston theory! 
apparatus of the planar, vertical type, turbulence commences at a value of the Reynolds number of about 25. Often it is clear that $\operatorname{Re}<<25$. If there is uncertainty, the $\Delta t$ for transition from laminar to turbulent flow should be determined experimentally. At constant pressure and constant average gas temperature, eq (8) indicates that $\dot{q} / \Delta t$ versus $\Delta t$ is linear for laminar flow. By straight line extrapolation of $\dot{q} / \Delta t$ versus $\Delta t$ to zero $\Delta t$, the heat transport by convection is eliminated.

The essential requirements of eq (8) are confirmed by the results. The heat transport correlates well versus $\frac{\alpha \rho^{2} c_{p} \Delta t}{\eta}$. The $\dot{q} / \Delta t$ versus $\Delta t$ extrapolations are linear within the precision of the data, except for very large values of eq (8) where second order effects may be detectible. The values of $\Delta t$ calculated from eq (10) (indicated by an asterisk on some extrapolation isotherms in the next paper) are in satisfactory accord with the experimental measurements.

\section{Asymmetry of the Heat Flow}

\subsection{Coaxial Centering}

If the emitter is not centered perfectly the geometrical factor of $\ln r_{2} / r_{1}$ should be replaced by $\cosh ^{-1}\left[\left(r_{2}^{2}+r_{1}^{2}-d^{2}\right) / 2 r_{1} r_{2}\right]$, where $d$ represents the displacement of the two axes. For the cell of figure 1 , an error of $7 \times 10^{-4} \mathrm{~cm}$ in the centering would make a difference of only one part in 100,000 in the cell constant.

\subsection{Heat Loss From the Bottom of the Emitter}

The cell with a single heat guard loses heat on the bottom as well as on the sides. With a uniform heater winding this leads to an asymmetric temperature distribution, which becomes larger as the gas thermal conductivity becomes larger. A reasonable approximation permitting mathematical treatment assumes uniform heat flow from the center heater to the emitter, no heat flow on the heat guard end, and the heat flow across the conductivity gap proportional to the temperature difference. The solution for this problem is

$$
-K_{\mathrm{Ag}} \Delta t / 2 \dot{q}=h \sum \frac{ \pm \sqrt{\alpha_{n}^{2}+h^{2}} \cos \left(\alpha_{n} z\right) \phi_{0}(r ; n)}{\alpha_{n}^{2}\left(\left[\alpha_{n}^{2}+h^{2}\right] l+h\right) \phi_{1}(a ; n)}
$$

where $h=A K, A$ is the total exterior surface area, $K$ is the thermal conductivity of the substance in the gap, and $K_{\mathrm{Ag}}$ the thermal conductivity of silver. ${ }^{5}$

$$
\begin{gathered}
a \leq r \leq b \\
0 \leq z \leq l \\
\phi_{0}(r ; n)=I_{0}\left(r \alpha_{n}\right)\left[\alpha_{n} K_{1}\left(b \alpha_{n}\right)-h K_{0}\left(b \alpha_{n}\right)\right] \\
+K_{0}\left(r \alpha_{n}\right)\left[\alpha_{n} I_{1}\left(b \alpha_{n}\right)+h I_{0}\left(b \alpha_{n}\right)\right]
\end{gathered}
$$

${ }^{5}$ If the cell were made of another material, the corresponding thermal conductivity would be used in place of $K_{\mathrm{Ag} \text {. }}$

$$
\begin{aligned}
\alpha_{n} \phi_{1}(a ; n)= & \left(\frac{\partial \phi_{0}}{\partial r}\right)_{r=a} \\
= & I_{1}\left(a \alpha_{n}\right)\left[\alpha_{n} K_{1}\left(b \alpha_{n}\right)-h K_{0}\left(b \alpha_{n}\right)\right] \\
& \quad-K_{1}\left(a \alpha_{n}\right)\left[\alpha_{n} I_{1}\left(b \alpha_{n}\right)+h I_{0}\left(b \alpha_{n}\right)\right]
\end{aligned}
$$

and the $\alpha_{n}$ are the ordered positive roots of $h=\alpha_{n} \tan \left(\alpha_{n} l\right)$. The value of $\sqrt{\alpha_{n}^{2}+h^{2}}$ is taken positive or negative in accord with the sign of $\sin \alpha_{n} l$.

The functions $I_{0}, I_{1}, K_{0}$, and $K_{1}$ are Bessel functions of complex argument. The result shows that the average temperature of the emitter is not at the center but at

$$
\begin{aligned}
z & =0.66 l \text { for } K=5.95 \times 10^{-5}, \\
z & =0.675 l \text { for } K=5.95 \times 10^{-4}, \\
\text { and } z & =0.697 l \text { for } K=11.90 \times 10^{-4} .
\end{aligned}
$$

Conductivities calculated from the average of the top and center temperatures must be increased by the factor indicated in figure 4 . This factor is accurate enough that usually no significant error is involved, and could be reduced by adding measurement of the temperature difference at the bottom of the cell.

\section{The "Blank" and Conduction of Pins}

When the cell is evacuated, the heat transferred by radiation and by conduction of the pins can be treated as a measurement of a "conductivity," called a "blank." The present cell had a blank at $0{ }^{\circ} \mathrm{C}$ of $0.138 \times 10^{-5} \mathrm{cal} \mathrm{cm}^{-1} \mathrm{sec}^{-1} \mathrm{deg} \mathrm{C}^{-1}$, about 4 percent of the conductivity of $\mathrm{CO}_{2}$ at the same temperature and $1 \mathrm{~atm}$ pressure. It was estimated that no more than half of the heat transfer of that blank was due to conduction of the pins.

It was assumed that the conductivity of a gas could be found by deducting the blank from the value of the apparent conductivity for zero temperature difference. However, the blank may not adequately represent the heat transfer in the presence of a gas for the following reasons:

(1) The conduction of heat across the pin-emitter interface may increase in the presence of a gas.

(2) The temperature distribution in the pins may perturb the heat transfer in the gas significantly.

In the first case, if the pressure of the pins against the emitter is high enough, the effect of the gas on the conduction across the contact interface is negligible. Ascoli and Germagnoli [5] showed that the temperature difference across a steel-aluminum interface with $8 \mu \mathrm{in}$. finish became nearly constant in a vacuum once the pressure of contact reached 100 $\mathrm{kg} / \mathrm{cm}^{2}$. For $\mathrm{N}_{2}$ at $1 \mathrm{~atm}$, the temperature difference across the interface with the same heat flux became nearly constant at $50 \mathrm{~kg} / \mathrm{cm}^{2}$ contact pressure, and at $100 \mathrm{~kg} / \mathrm{cm}^{2}$ was equal within experimental error to the corresponding vacuum value. Boeschoten and Van Der Held [6] found three times the heat conduction in the presence of $1 \mathrm{~atm}$ of helium as in a vacuum at $35 \mathrm{~kg} / \mathrm{cm}^{2}$ contact pressure. The inference from the preceding investigation is that at $100 \mathrm{~kg} / \mathrm{cm}^{2}$, 
the ratio would be $1.5 / 1$. Rough surfaces approach the behavior of smooth surfaces as the contact pressure is increased. These measurements were performed upon a steel-aluminum interface rather than a Pyrex-silver interface used in the present apparatus. However, the ability of silver to conform to the harder surface of Pyrex is at least as great as for aluminum to conform to steel. The pins were forced into the silver sufficiently that indentations 0.003 to $0.005 \mathrm{in}$. deep were made in the emitter, which is to say with sufficient force to exceed $1500 \mathrm{~kg} / \mathrm{cm}^{2}$, the tensile strength of silver. The Pyrex pins were held in an aluminum sleeve such that the composite expansion of the two materials approximately matches the expansion of the cell material from 0 to $400{ }^{\circ} \mathrm{C}$. Consequently the centering of the emitter and the contact pressure between the pin and the emitter should be maintained over the temperature range. Hence it can be expected that gases of low conductivity caused an insignificant change in the rate of transfer of heat through the pins. In helium, the uncertainty in pin conduction should not exceed 0.1 percent of the gas conduction.

The additional heat transfer through the gas, which arises because the temperature distribution in the pins does not follow the radial gradient, can be evaluated by relaxation methods, and leads to the result that an 18 percent increase of conduction for the area of the surface occupied by the base of the pin will be observed when the conductivity of the gas is 10 percent that of Pyrex. The area occupied by the bases of seven pins was 0.4 percent of the total surface area of the receiver so that at a gas conductivity of $3 \times 10^{-4} \mathrm{cal} \mathrm{cm}^{-1} \mathrm{sec}^{-1} \mathrm{deg} \mathrm{C}^{-1}$ an increase of conduction of 0.07 percent will be observed. If the conductivity of the fluid reached the value of Pyrex, the pins would have no effect on the temperature gradient, and at low values of gas conductivity, the effect is about the same as at a conductivity of $3 \times 10^{-4} \mathrm{cal} \mathrm{cm}^{-1} \mathrm{sec}^{-1} \mathrm{deg} \mathrm{C}^{-1}$.

\section{Radial Temperature Gradient in the Emitter and Receiver}

A correction, in general small, must be made for the fact that the thermocouples are placed in the body of the emitter and receiver. This is a power series of $K_{\text {corr }}=K_{\text {meas }}\left[1+a K_{\text {meas }}+\left(a K_{\text {meas }}\right)^{2}+\right.$. . . . of which only the first corrective term is large enough to be significant. For the dimensions of the silver conductivity cell in figure $1, a=10.70 \mathrm{~cm}$ sec deg $\mathrm{C} / \mathrm{cal}$.

\section{Discussion of Other Cells}

There are only a few forms of thermal conductivity cells suitable for accurate absolute determinations of the thermal conductivity of gases. The "hot wire" cell is an extreme case of a coaxial cylinder cell, with a small radius of the emitter, but the cell constant cannot be determined with the accuracy possible for a larger radius of emitter. The heat transfer analysis is complicated, and in a dense gas convection is difficult to control because of the large temperature gradient near the wire.

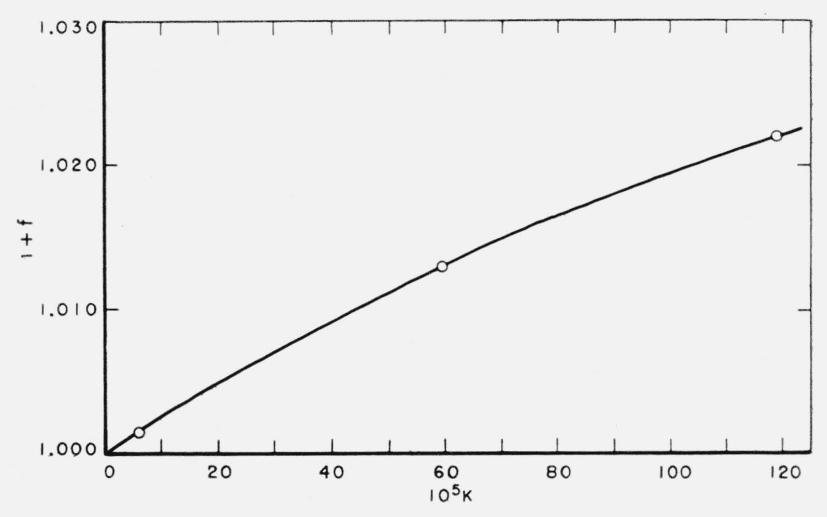

FIguRE 4. Correction to the conductivity for asymmetric heat flow.

A coaxial cylinder cell with heat guards at both ends would not have the end heat flow which requires the corrections given in figure 4 . However, there is an uncertainty in the results due to a possible heat flow between the emitter and heat guards. When the emitter is suspended or held in place by sleeve or rod connections with the heat guards (as is customary for this design), the increased conduction possible by a slight difference in temperature between the emitter and guards will increase significantly the uncertainty of the thermal conductivity determination. The uncertainty is reduced if the thermal conductivity is relatively large, and if the emitter-receiver temperature difference is large. In order to avoid these restrictions, the suspension method could probably be modified.

In principle the flat plate cell, with an emitter and guard above the receiver, should not be affected by convection. In dense gas, there is good possibility that many such cells have had significant convection transfer from "chimneys," but that no special measurements were made to check. However, by proper design, it can be expected that convection difficulties would be eliminated. The cell constant can be determined with about the same accuracy as for the coaxial cylinder cell, but instability of the flat plate cell alinement affects the cell constant directly. By contrast, a change of the coaxial cylinder cell alinement affects the cell constant comparatively little.

\section{Conclusions}

By use of a heat guard and receiver extension which extends the conductivity gap of a coaxial cylinder, the conduction across the gap can be expressed accurately. In combination with special measuring techniques involving a "falling probe," the value of the cell constant can be obtained to an accuracy within \pm 0.1 percent.

The coaxial cylinder cell must be used with the axis vertical in order to permit analysis of convective heat transport. It was deduced mathematically and found experimentally that the apparent conductivity is affected by laminar convection linearly with the temperature difference. The temperature difference which causes the Reynolds number, eq (10), to be 
25 defines the highest temperature difference for which laminar flow can be expected. The effects of convection can be eliminated by extrapolating the apparent conductivity versus the temperature difference to zero temperature difference, provided the measurements lie in the range of laminar flow.

For high accuracy, corrections must be made to the data for asymmetry of heat flow, perturbation of the temperature gradient by the mounting pins, and the temperature gradient in the emitter and receiver. Of course, the rate of heat transfer in a vacuum is deducted. There has now been sufficient quantitative measurement to show that the radiation and pin transfer should remain constant within 0.1 percent in the presence of a gas.

Of the various types of cells used for measuring the thermal conductivity of gases, the coaxial cylinder cell and the flat plate cell offer the most promise of accurate results.

\section{References}

[1] H. S. Carslaw and J. C. Jaeger, Conduction of heat in solids, 2d Ed., Oxford Univ. Press, London, 1959.

[2] W. H. MacAdams, Heat transmission, 2d Ed., McGrawHill Book Co., Inc., New York, 1942.

[3] R. C. Jones and W. H. Furry, Rev. Mod. Phys. 18, 151 (1946).

[4] L. Onsager and W. W. Watson, Phys. Rev. 56, 474 (1939).

[5] A. Ascoli and E. Germagnoli, Energia Nucleare 3, 113 (1956).

[6] F. Boeschoten and E. F. M. Van Der Held, Physica 23, 37 (1957).

\section{Appendix}

Given the conductivity gap $h$ and heat guard gap $2 k{ }^{6}$ We identify the points, $A B C D E F G H$ in the complex $z$-plane. These points are transformed in the $t$-plane by the Schwarz-Christoffel transformation, which is

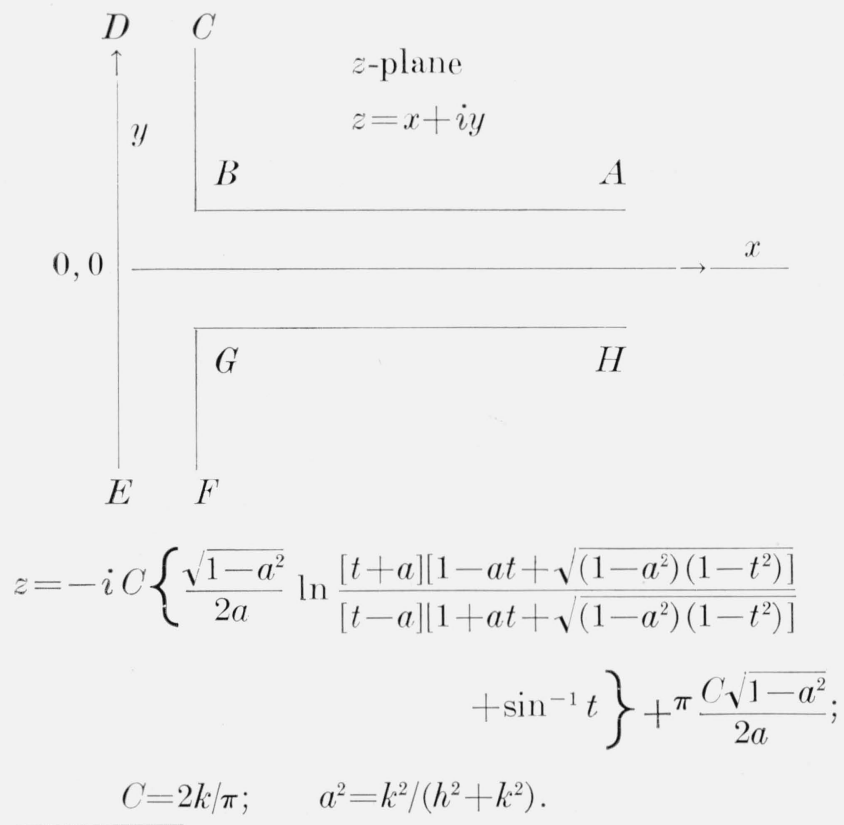

${ }^{6}$ Note that the symbolism is that of Carslaw and Jaeger, and in general should not be identified with the symbolism of the rest of the paper.
Points $A, H, C, D, E$, and $F$ are assumed to lie at infinity in the z-plane. Corresponding values of the points are shown for the $t$-plane.

$$
\begin{aligned}
& t \text {-plane } \\
& t=\zeta+i \xi \\
& \begin{array}{cccc|cccc}
A & B & C, D & 0, & 0 & E, F & G & H \\
\hline-\infty & -1 & -a & 0 & 0 & a & 1 & \infty
\end{array}
\end{aligned}
$$

By use of the conformal mapping function

$$
\pi w=\ln (t+a) /(t-a)
$$

the points in the z-plane are transformed to the indicated points in the $w$-plane.

\begin{tabular}{lr|lr}
$\begin{array}{l}w \text {-plane } \\
w=\mu+i v\end{array}$ & \\
$C$ & $A$, & $H$ & $F$ \\
\hline$-\infty$ & 0 & 0 & $\infty$ \\
$D$ & 0 & 0 & $E$ \\
\hline$-\infty-i$ & 0, & $-i$ & $\infty,-i$
\end{tabular}

Thus in the $w$-plane, heat flow between two infinite parallel plates can be studied.

If the heat flow to $w=u-i$ is studied, this corresponds to the heat flow to $D E=-i y$ in the z-plane. By substituting $\pi w=\ln \left(\frac{t+a}{t-a}\right)$ and $w=u-i, z=-i y$,

$$
\begin{array}{r}
-i y=-i h(u-i)-\frac{i h}{\pi} \ln \frac{\left[1-a t+\sqrt{\left.\left(1-a^{2}\right)\left(1-t^{2}\right)\right]}\right.}{\left[1+a t+\sqrt{\left(1-a^{2}\right)\left(1-t^{2}\right)}\right]} \\
-\frac{2 i k}{\pi} \sin ^{-1} t+h .
\end{array}
$$

For $u$ (and $y$ ) large, $t \rightarrow+a^{-}$

$$
y=h u+\frac{h}{\pi} \ln \left(1-a^{2}\right)+\frac{2 k}{\pi} \sin ^{-1} a .
$$

The heat transport to $D E$ per unit width per unit temperature difference is, in the $w$-plane, $K u$ (where $K$ is the thermal conductivity of the medium).

$$
K u=K\left(\frac{y}{h}-\frac{1}{\pi} \ln \left[1-a^{2}\right]-\frac{2 k}{\pi h} \sin ^{-1} a\right) .
$$

Thus, there are two corrective terms to be added to the normal term $y / h$ for the steady heat flow between two planes distant $h$ apart. For the case that the heat guard gap $(2 k)$ is equal to the conductivity gap $(h)$

$$
a^{2}=1 / 5
$$


Where the conduction is regarded as unperturbed half the heat guard gap times the factor in parenalong $F G$ and $B C$, we can get a relative correction to the normal term for infinite plates over the distance of the heat guard gap by taking $y=2 k=h$. thesis, i.e.,

Then

$$
K u=K\left(1-\frac{1}{\pi} \ln \left[1-a^{2}\right]-\frac{1}{\pi} \sin ^{-1} a\right)
$$

$$
\begin{aligned}
c_{2} & =k\left(1-\frac{1}{\pi} \ln \left[1-a^{2}\right]-\frac{1}{\pi} \sin ^{-1} a\right) \\
& =k(1-0.0766)=0.9234 k .
\end{aligned}
$$

or we may add a length to the emitter equal to one

(Paper 66A4-168) 